\title{
Sensory Stimulation Training for BCI System based on Somatosensory Attentional Orientation
}

\author{
Lin Yao, Xinjun Sheng, Natalie Mrachacz-Kersting, \\ Xiangyang Zhu, Dario Farina, Fellow, IEEE, Ning Jiang, Senior Member, IEEE
}

\begin{abstract}
In this study, we propose a sensory stimulation training (SST) approach to improve the performance of a braincomputer interface (BCI) based on somatosensory attentional orientation (SAO). In this BCI, subjects imagine the tactile sensation and maintain the attention on the corresponding hand as if there was a tactile stimulus on the wrist skin. Twenty BCI naïve subjects were recruited and randomly divided into a Control-Group and an SST-Group. In the Control-Group, subjects performed left hand and right hand SAO tasks in six consecutive runs (with 40 trials in each run), divided into three blocks with each having two runs. For the SST-Group, two runs included real tactile stimulation to the left or right hand (SST training block), between the first two (Pre-SST block) and the last two SAO runs (Post-SST block). Results showed that the SSTGroup had a significantly improved performance of $9.4 \%$ between the last block and the first block after SST training $\left(\mathbf{F}_{(2,18)}=11.11\right.$, $p=0.0007)$; in contrast, no significant difference was found in the Control-Group between the first, second and the last block $\left(\mathbf{F}_{(2,18)}\right.$ $=2.07, p=0.1546$ ), indicating no learning effect. The tactile sensation-induced oscillatory dynamics were similar to those induced by SAO. In the SST-Group, $\mathbf{R}^{2}$ discriminative information was enhanced around the somatosensory cortex due to the real sensory stimulation as compared with that in the Control-Group. Since the SAO mental task is inherently an internal process, the proposed SST method is meant as an adjuvant to SAO to facilitate subjects in achieving an initial SAObased BCI control.
\end{abstract}

\section{INTRODUCTION}

A brain-computer interface (BCI) provides a direct interaction between the brain and the external environment, which would be particularly useful for locked-in patients [1]. Without the requirement of external stimuli, patients and healthy people can use sensory-motor rhythm (SMR) based BCIs by mentally performing motor imagery (MI) of their left or right hand [2][5]. The MI-induced event-related desynchronization (ERD)/ synchronization (ERS) brain signals [6], [7] and movement related cortical potentials (MRCP) [8], [9] enable direct BCI control without any external stimuli, and have attracted extensive interests [10]-[13]. Independent BCIs have a wide range of potential applications, such as wheelchair control [14], [15], helicopter navigation [16], [17], robotic arm control [18],

Lin Yao, and Ning Jiang are with Department of Systems Design Engineering, Faculty of Engineering, University of Waterloo, Waterloo,

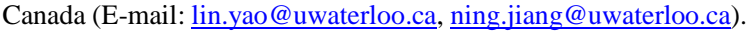

Xinjun Sheng and Xiangyang Zhu are with State Key Lab of Mechanical System and Vibration, Shanghai Jiao Tong University, Shanghai, China. (xjsheng@sjtu.edu.cn, mexyzhu@sjtu.edu.cn).

Natalie Mrachacz-Kersting is with Center for Sensory-Motor Interaction, the Faculty of Medicine, Aalborg University, Aalborg, Denmark. (nm@hst.aau.dk)

Dario Farina is with Department of Bioengineering, Imperial College London, London, UK. (d.farina@imperial.ac.uk) and neuro-prostheses in patients suffering from a high level spinal cord injury [19], [20], and for motor function rehabilitation of stroke patients [21]-[24]. Complementary to motor imagery detection, we proposed a different type of SMRbased BCI. Rather than focusing on oscillatory dynamics of motor-related cortical activities, we demonstrated that oscillatory dynamics induced by tactile stimuli delivered to different parts of the body allow reliable decoding of the subject's intentions [25]-[27]. In this new somatosensory BCI, the proposed tactile selective sensation (SS) system significantly improves the current tactile BCI performance reported in the literature [28]-[31]. Further, the imagined sensation intentions, or sensory imagery (SI) can also be decoded from the spontaneous EEG rhythm. We demonstrated the feasibility of an independent BCI based on specific SI tasks, in which subjects imagined tactile stimulation coming from different locations of the body, and we named this as somatosensory attention orientation (SAO) [32]. SAO provides new strategies for independent BCIs, with the benefit of increasing BCI diversities in a stimulus-independent BCI framework [32], [33].

In order to make oscillatory BCI more applicable (including both motor and somatosensory BCI modalities [34]), intensive research efforts have been conducted to further improve MIbased BCI performance, and also to reduce the number of BCIilliterate users. Machine learning algorithms for detection of MI have been largely improved, including pre-processing by spatial filtering, such as the Common Spatial Pattern (CSP) [11], [12]. However, an approximately 5\% improvement was reported on average across datasets when more advanced algorithms, such as optimized spatial-spectrum filtering based on mutual information, were implemented [13]. Additionally, some users were still unable to attain the acceptable $70 \%$ accuracy, even with state-of-the-art algorithms [35], [36]. Subject training has an important role on BCI performance [16], [37]. Neurofeedback-based training has received extensive interest [38]-[41], establishing a framework of how to train subjects to gain sufficient BCI control. Nonetheless, even after several training sessions, some individuals still remain BCIilliterate as their BCI performance was below the acceptable level (70\% accuracy for a two-state system) [35], [36]. Machine learning algorithms have been developed to extract subjectspecific patterns, so as to improve the performance of individuals. Subjective training approaches to modulate rhythmic activity have also been explored for this purpose [16]. In addition, the coadaptation of the subject and the machine learning has also been shown to reduce the number of poorly performing BCI users to some extent [42]. Recently, a new training approach that may facilitate MI decoding by utilizing 
somatic stimulation for calibration and guiding subjects has been reported [43]. This system comprises a wrist vibration device, which passively produces stimulus-induced ERD/ERS dynamics, similar to those induced by MI. It is likely that these MI-improving efforts would lead to similar improvements of the somatosensory based BCI system, since SAO-induced oscillatory dynamics are highly similar to those induced by MI [33]. Since SS and SAO are both encoded in the somatosensory system, the sensory stimulation likely provides an even more intuitive way for users to learn a SAO-based BCI control.

Due to the similarity among SS-induced [27], [32] and SAOinduced oscillatory dynamics [32], [33], sensory stimulation would provide a novel way to train subjects to achieve an initial SAO-BCI control. In our recent study, we have shown that subject's covert somatosensory attention can be reliably decoded from a BCI system calibrated with tactile sensation [44], indicating the high similarity in EEG from SS and SAO tasks. Therefore, we hypothesized that the real sensory stimulation would provide a sensory guidance to help the subject to perform SAO tasks. Therefore, we expected that there would be an SAO-BCI performance improvement after actual sensory stimulation. In the current study, we tested this hypothesis by using sensory stimulation training (SST) to improve SAO performance in healthy subjects.

\section{METHODS}

\section{A. Subjects}

Twenty healthy BCI naïve subjects were recruited in the experiments (10 females, all right-handed, average age $24.2 \pm 2.2$ years). Subjects were randomly divided into two groups, i.e. sensory stimulation training group (SST-Group) and control group (Control-Group), with ten subjects in each group. The study was approved by the Ethics Review Board of the University of Waterloo, Waterloo, Canada (ORE\#: 22295). An informed consent form was signed by all participants before participation.

\section{B. EEG Recordings and Somatosensory Stimulation}

EEG signals were recorded using a 32-channel wireless g.Nautilus EEG system (g.tec, Austria). The electrodes were placed in accordance to 10-10 system. The reference electrode and ground electrode were placed on the right earlobe and the forehead, respectively. EEG signals were digitally sampled at $250 \mathrm{~Hz}$.

The dorsal lateral side of the wrists was mechanical stimulated, using linear resonant actuators $(10 \mathrm{~mm}, \mathrm{C} 10-100$, Precision Microdrives Ltd., typical normalized amplitude 1.4 G). The actuator was set to produce a $27-\mathrm{Hz}$ sine wave stimulus, which was modulated with a $175-\mathrm{Hz}$ sine carrier wave. This type of stimulus in known to active both the Pacinian and Meissner corpuscles [45]. The optimal amplitude was adjusted based on individual feedback, such that they were comfortable with perceiving the vibration [27], [44].

\section{Experiment Paradigm}

The overall structure of the experimental protocols of the two groups were similar. Each session comprised three blocks. Each

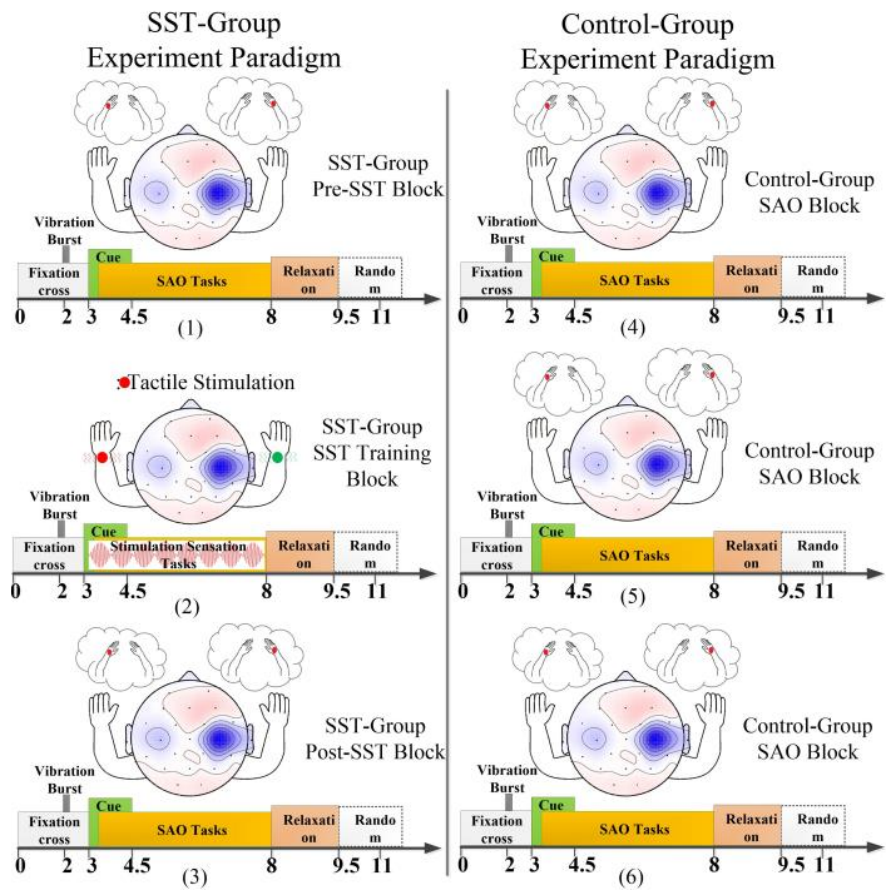

Figure 1. Graphic Illustration of the experimental paradigm in SST-Group (1-3) and Control-Group (4-6). (1) Block one of SST-Group protocol had two runs, during which subjects performed left and right hand SAO tasks. (2) Block two of SST-Group protocol had two runs, during which subjects performed the real sensation tasks when left or right hand wrist was tactile stimulated; (3) Block three of SST-Group protocol had two runs, during which subjects performed left and right hand SAO tasks the same as in block one. (4), (5) and (6) Control-Group protocol, subjects only performed left and right hand SAO tasks in all six runs.

block included two experimental runs, with continuous EEG recordings. Short breaks were provided to the subject between two runs to avoid mental fatigue and habituation. Additional resting periods $(5-10 \mathrm{~min})$ were also provided between the blocks. In each run, the subject would perform 40 mental tasks. The differences between the two protocols are outlined below: 1) SST-Group Protocol

The SST-Group Protocol is illustrated in Fig. 1(1)-(3), for the three blocks.

Pre-SST Block (Run 1 to Run 2): During the experiment, the subjects were required to limit their eye blinks, facial and arm movement. At the beginning of each trial $(\mathrm{T}=0 \mathrm{~s})$, a white fixation cross ("“") appeared in the center of the screen. At T= $2 \mathrm{~s}$, a vibration pulse was applied to both wrists for $200 \mathrm{~ms}$ to alert the user to be ready for the task. At the 3rd second ( $\mathrm{T}=3$ $\mathrm{s}$ ), a red cue bar pointing either to the left or right was randomly presented: 1) left corresponded to the SAO-L task, during which the subject shifted and maintained the somatosensory attention on the left wrist, and imagined the tactile sensation even when there were no tactile stimuli; 2) right corresponded to the SAO-R task. This cue lasted for $1.5 \mathrm{~s}$ and then disappeared. The SAO task continued for $5 \mathrm{~s}$, until the fixation symbol disappeared $(\mathrm{T}=8 \mathrm{~s})$. This was followed by a $1.5 \mathrm{~s}$ relaxation period. Finally, to limit subject adaptation, a random time of $0 \sim 2 \mathrm{~s}$ was appended to the relaxation period. A total of 80 trials ( 40 trials for each task) were performed by the subjects in 2 runs, in a randomized order.

SST Training Block (Run 3 to Run 4): During this block, 
the timing of the trial and the cues the subject received were the same as for block one. The difference was the task to be performed. When the red cue bar was either presented to the left or the right side, the tasks to be performed were: 1) SS-L task, in which the subject should focus on the sensation when the left wrist was stimulated; 2) SS-R task, in which the subject should focus on the sensation when the right wrist was stimulated. In each trial, the tactile sensation continued for $5 \mathrm{~s}$, until the fixation symbol disappeared. A total of 80 trials (40 trials for each task) were performed by the subjects in 2 runs, in a randomized order.

Post-SST Block (Run 5 to Run 6): Block three was identical to Block one.

\section{2) Control-Group Protocol}

The experimental protocol of the Control-Group is illustrated in Fig. 1 (4)-(6). The three blocks were identical to Block One of the SST-Group, in which the subjects were asked to perform either SAO-L or SAO-R tasks. Therefore, 120 trials for SAO-L and SAO-R (240 trials in total) were performed in six runs (three blocks).

\section{Algorithms and Performance Evaluation}

The Common Spatial Pattern (CSP) algorithm was adopted to enhance the feature discrimination among the investigated tasks [46], [47]. The log-variances of the first and last three CSP components were chosen as feature vectors, and linear discriminative analysis (LDA) for classification. The analyzed frequency bands were [27]: alpha-beta [8 26] Hz $(\alpha \beta)$, beta [13 26] $\mathrm{Hz}(\beta)$, alpha [8 13] $\mathrm{Hz}(\alpha)$, lower beta $\left[\begin{array}{ll}13 & 20\end{array}\right] \mathrm{Hz}(\beta-)$, upper beta $\left[\begin{array}{ll}20 & 26\end{array}\right] \mathrm{Hz}(\beta+)$, lower alpha $\left[\begin{array}{ll}8 & 10\end{array}\right] \mathrm{Hz}(\alpha-)$, upper alpha $\left[\begin{array}{ll}10 & 13\end{array}\right] \mathrm{Hz}(\alpha+)$, and eta $\left[\begin{array}{ll}10 & 16\end{array}\right] \mathrm{Hz}(\eta)$. Before the CSP spatial filtering, a fourth-order Butterworth filter was applied to the raw EEG signals. A 10-fold cross-validation on data from every subject was used to evaluate the offline BCI performance, and for selection of subject-specific frequency bands.

EEG signals from 1 to $4 \mathrm{~s}$ with respect to the appearance of the red cue bar were segmented. In the SST-Group, the data of

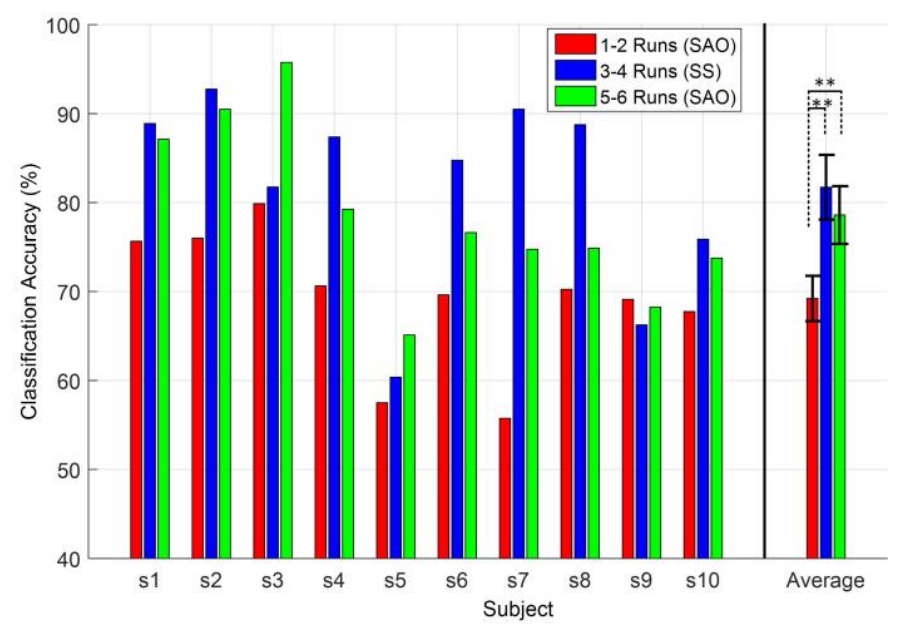

Figure 2. BCI performance during the first, second and last block in the SST-Group, with two runs in each block. Note: the red bar indicates the performance of the first block (Pre-SST block); the blue bar the performance of the second block (SST training block); and the green bar the performance of the last block (Post-SST block); two stars indicate significance at $\mathrm{p}<0.01$. the first block were extracted for the evaluation of the pre-SST SAO performance; the data of the last block were extracted for the evaluation of the post-SST SAO performance; the data of the second block were also extracted to evaluate performance of the stimulus-induced oscillatory pattern (SST-induced EEG dynamics). Correspondingly, in the Control-Group the data of the first, second, and last block were extracted for performance evaluation.

\section{E. Calculation of EEG Dynamics and Time-Frequency Decomposition}

ERD (ERS) is defined as the percentage of power decrease (increase) in a defined frequency band with respect to a reference interval (e.g. resting state before the task) [48]. The [8 26] $\mathrm{Hz}(\alpha \beta)$ frequency band was adopted in this study for EEG filtering prior to the ERD/ERS calculation. The reference interval was chosen from $1.2 \mathrm{~s}$ to $2.0 \mathrm{~s}$ prior to the appearance of the red cue bar.

The area formed by C3 and C4 ERD/ERS curves between 1 and $4 \mathrm{~s}$ (after the appearance of the red cue bar) represents the hemisphere difference (HD) as induced by the task, i.e. the area enclosed by the curve of $\mathrm{C} 4-\mathrm{C} 3$ and between 1 to $4 \mathrm{~s}$ was defined as HD index. The HD difference (HDD) between the left and right task was further used for comparison between different blocks.

The EEGLAB toolbox was used to manually correct for artifacts in the EEG signal [49], and trials contaminated by artefacts such as due to swallowing and movement were excluded. Time-frequency decomposition was applied to each EEG channel. This was calculated every $200 \mathrm{~ms}$ with a Hanning tapper, convoluted with a modified sinusoid basis, in which the number of cycles linearly changed with frequency to achieve proper time and frequency resolution [50]. The $\mathrm{R}^{2}$ index [51], [52] was defined as the squared Pearson-correlation coefficient between feature and class label. Accordingly, the $\mathrm{R}^{2}$ was calculated in the spatio-spectral-temporal domain and was used to locate the component of different EEG channels for the classification of the corresponding mental tasks.

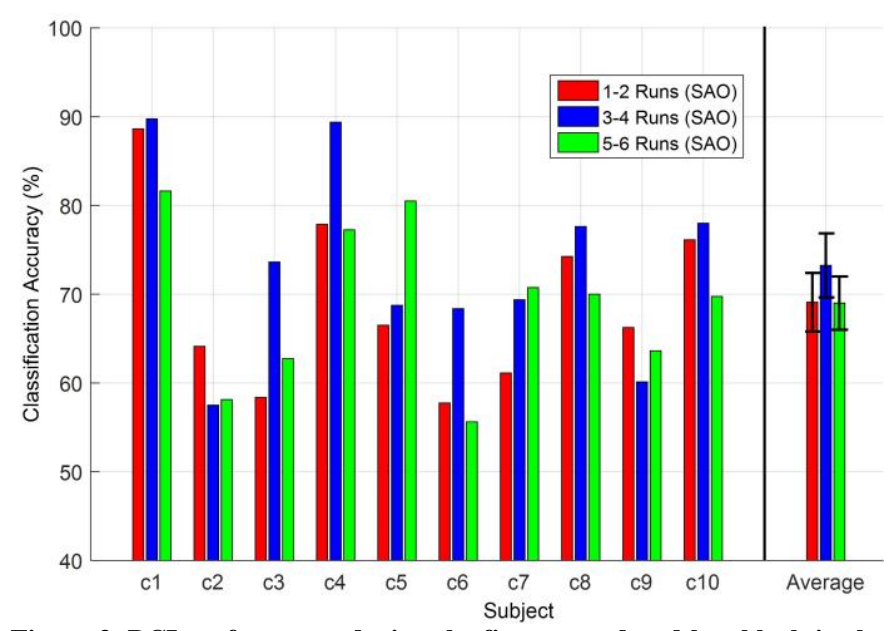

Figure 3. BCI performance during the first, second and last block in the Control-Group, with two runs in each block. Note: the red bar indicates the performance of the first block; the blue bar the performance of the second block; and the green bar the performance of the last block. 


\section{F. Statistics}

One-way ANOVA with repeated-measures was used to analyze performance differences among different experimental blocks (with $p=0.05$ ). Whenever the main effect was found to be significant, Bonferroni correction based on multiple comparisons was used for post-hoc testing.

\section{RESULTS}

Fig. 2 illustrates the BCI performance of the first, second and the last block for the SST-Group. One-way ANOVA with repeated measures showed that there was a significant difference in classification accuracy among the three blocks (Pre-SST, SST, Post-SST; $\left.\mathrm{F}_{(2,18)}=11.11, p=0.0007\right)$. Post-hoc comparison $(p=0.05)$ showed that the performance of the postSST block (78.6 \pm 9.7$)$ was significantly greater than that of the pre-SST block (69.2 \pm 7.6$)$. The classification of the SST block $(81.7 \pm 10.9)$ was also significantly higher than that of the first block. No significant difference was found between the SST and post-SST block.

Fig. 3 illustrates the BCI performance of the first, second and the last block for the Control-Group. No significant difference in classification accuracy was found among the first $(69.1 \pm 10.0)$, the second $(73.3 \pm 10.8)$ and the last block $\left(69.0 \pm 9.0 ; \mathrm{F}_{(2,18)}=2.07, \mathrm{p}=0.1546\right)$. Moreover, the performance of the first Block from both groups were similar $(69.0 \%$ on average for the Control-Group, 69.2\% for the SST-Group).

Fig. 4 shows the grand-averaged oscillatory dynamics ([826] $\mathrm{Hz}$ ) across SAO and SS tasks in different experimental phases for the SST-Group. At the -1s, a vibration burst resulted in a clear power reduction (ERD) with the same strength within the alpha-beta frequency for both $\mathrm{C} 3$ and $\mathrm{C} 4$ for all tasks and all phases (the $\mathrm{C} 3$ and $\mathrm{C} 4$ channels are shown since they correspond to the sensory motor regions). During the time

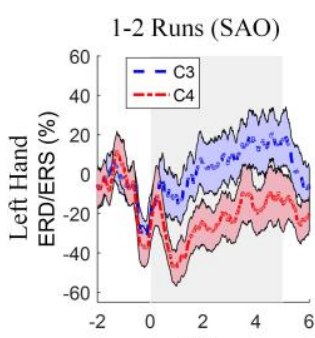

(1)

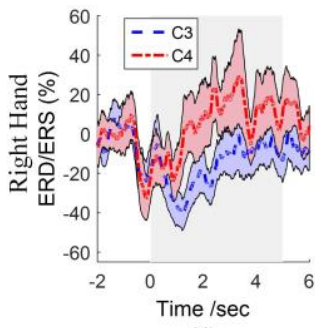

(4)

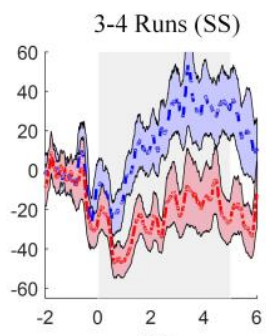

(2)

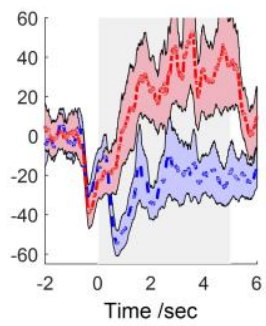

(5)

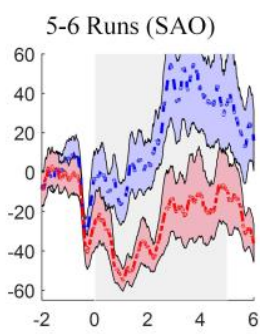

(3)

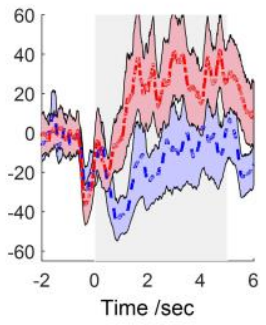

(6)
Figure 4. The time varying grand-averaged ERD/ERS curves at smallLaplace filtered $\mathrm{C} 3$ and $\mathrm{C} 4$ channels within the alpha-beta frequency band [8 26] Hz in the SST-Group. ERD/ERS for the (1) SAO-L task in the first block, (2) SS-L task in the second block, (3) SAO-L task in the last block; (4) SAO-R task in the first block, (5) SS-R task in the second block and (6) SAO-R task in the last block. The upper and lower curves indicate standard error. Time 0s corresponds to the time when the cue appeared (3rd second from the beginning of the trial). between $0 \mathrm{~s}$ to $5 \mathrm{~s}$ of the trials in the SST training blocks the sustained stimulation applied on the left (Fig. 4 (2)) or right wrists (Fig. 4 (5)) produced distinctive ERD/ERS dynamics across the left and right somatosensory cortex, i.e. clear contralateral ERDs. For SS-L tasks, the ERD was more pronounced on the right hemisphere while for the SS-R tasks, the ERD was stronger on the left hemisphere. Such ERD patterns are also evident for the SAO tasks in both the first and last block. Most importantly, such ERD dynamics are more distinctive between SAO-L and SAO-R in the third block (postSST) than in the first block (pre-SST). The ERD dynamics of the third block was indeed similar to that included by the second block, in which actual stimulation was applied. In contrast, the ERD dynamics of the Control-group were similar among the three blocks (Fig. 5). Unpaired t-test did not show significant differences in HDD in the first block between the SST-group and the Control-Group. The HDD was found to be significantly different among the three blocks in the SST-Group $\left(\mathrm{F}_{(2,18)}=4.96\right.$, $p=0.019)$. No significant difference was found in the ControlGroup $\left(\mathrm{F}_{(2,18)}=0.42, p=0.666\right)$. Post-hoc comparison $(\mathrm{p}=0.05)$ showed the HDD in the post-SST block was significantly higher than that in the pre-SST block. No significant difference was found between the SST and the post-SST block.

Furthermore, as different frequency band would have different ERD/ERS dynamics, the HDD in alpha ([8 13] Hz), low beta $([1320] \mathrm{Hz})$ and high beta $([2026] \mathrm{Hz})$ was tested in the SST-Group. No significant difference was found in alpha band $\left(\mathrm{F}_{(2,18)}=0.89, p=0.428\right)$. A significant difference was shown in both low beta and high beta $\left(\mathrm{F}_{(2,18)}=3.81, p=0.042\right.$ and $\mathrm{F}_{(2,18)}=4.68, p=0.023$ respectively). Post-hoc testing showed that the HDD of the post-SST block was significantly higher than that of the pre-SST block in low beta band, and the HDD of the SST block was significantly higher than that of the preSST block in the high beta band.

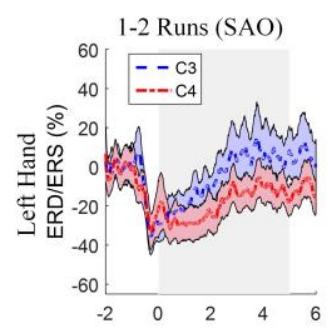

(1)

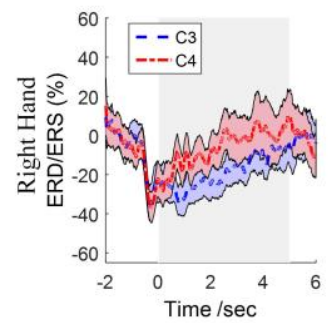

(4)

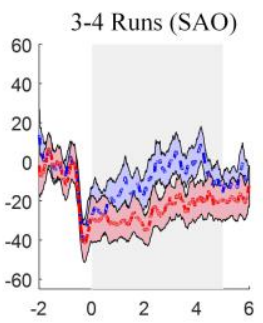

(2)

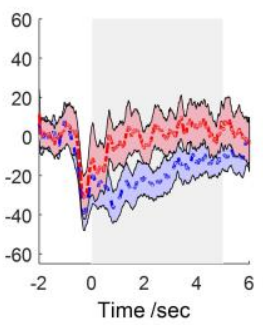

(5)

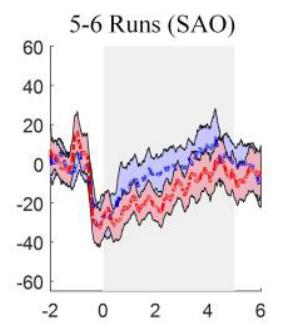

(3)

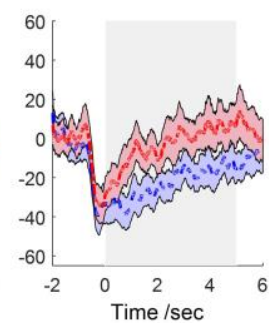

(6)
Figure 5. The time varying grand-averaged ERD/ERS curves at smallLaplace filtered $\mathrm{C} 3$ and $\mathrm{C} 4$ channels within the alpha-beta frequency band [8 26] Hz in the Control-Group. ERD/ERS for the (1) SAO-L task in the first block, (2) SS-L task in the second block, (3) SAO-L task in the last block, (4) SAO-R task in the first block, (5) SAO-R task in the second block and (6) SAO-R task in the last block. The upper and lower curves indicate standard error. Time 0 s corresponds to the time when the cue appeared (3rd second from the beginning of the trial). 


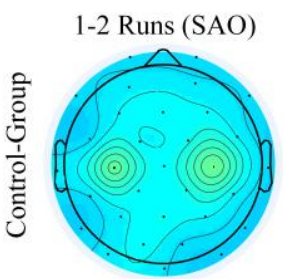

(1)

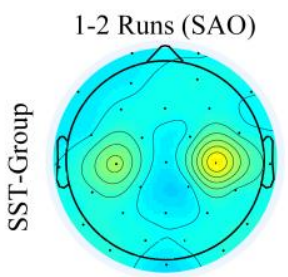

(4)

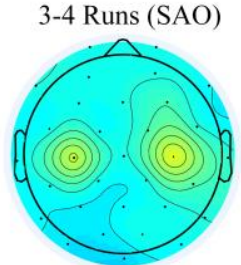

(2)

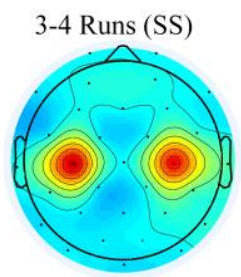

(5)

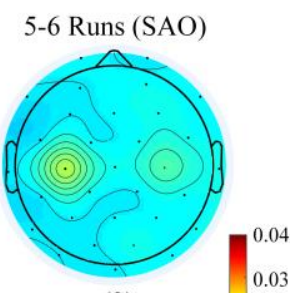

(3)

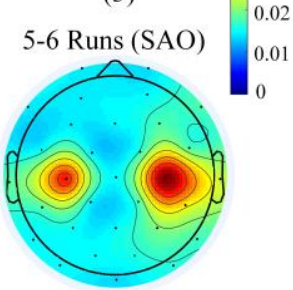

(6)
Figure 6. Grand-averaged $\mathbf{R}^{2}$ discriminative information distribution within the alpha-beta frequency band $\left[\begin{array}{ll}8 & 26\end{array}\right] \mathrm{Hz}$ across different runs period and group ( $R^{2}$ was averaged along the 1 to $4 \mathrm{~s}$ with respect to the appearance of the red cue bar). $R^{2}$ distribution with respect to (1) the first block in the Control-Group, (2) the second block in the Control-Group, (3) the last block in the Control-Group, (4) the first block in SST-Group, (5) the second block in SST-Group and (6) the last block in SST-Group. The color bar indicates the $\mathbf{R}^{2}$ value.

Fig. 6 illustrates the $\mathrm{R}^{2}$ discriminative information distribution across different blocks and groups. The discriminative information was mostly concentrated on left and right somatosensory cortex, which correlated with the results from the BCI performance analysis. For the Control-Group, no identifiable change was observed in the $\mathrm{R}^{2}$ values in the sensory cortex, across the three blocks. Conversely, for the SST-group, significant enhancement of $\mathrm{R}^{2}$ in the sensory cortex was observed from the first block to the last block.

\section{DISCUSSION}

In the current study, a novel sensory stimulation training method was proposed to facilitate sensory imagery decoding for SAO-based BCIs. To the best of our knowledge, this is the first time that sensory stimulation was introduced to improve the performance of BCIs based on covert somatosensory intentions. The SST-Group showed a $9.4 \%$ significant improvement in BCI classification accuracy when subjects received the tactile stimulation intervention. In contrast, the Control-Group showed no significant differences $(69.1 \%$ vs. $69 \%$ on average between the first and the third block). Moreover, the two groups showed similar initial SAO performance in the first block $(69.1 \%$ in Control-Group, $69.2 \%$ in SST-Group). Therefore, the observed improvement in BCI performance following SST intervention can only be explained by the intervention, rather than by the learning effect due to time involved in participation. The proposed SST training enhanced the $\mathrm{R}^{2}$ information especially in the somatosensory cortex (Fig. 6), reflecting a better SAO performance after SST training.

In order to exclude the effect of feedback-based training for improving SAO-based BCI control, no feedback was delivered to subjects after the mental task in both Control-Group and SST-Group. The only difference between the Control-Group and SST-Group was the second block, during which subjects in the Control-Group performed SAO tasks, while subjects in SST-Group received the real tactile stimulation. The grandaveraged ERD/ERS dynamics showed similarity among the first, second and the last block in the Control-Group, and no signicant performance difference was found, indicating no learning effect over time. By contrast, the ERD/ERS dynamics changed substantially in the SST-Group, especially in the second block that exhibited a higher ERS in the ipsilateral hemisphere. The ERS likely relates to the suppression of the activity, and the results presented here indicate that following the SST training, the ipsilateral suppression was enhanced. The ERD/ERS dynamics in the third block were similar to the SST training block, while greatly improved as compared with the first block. In both the Control and the SST-Group, the vibration burst on both hands consistently induced ERD changes, which were in accordance with our previous findings [27], [32], with co-activation of the left and right somatosensory cortex. Moreover, the oscillatory dynamics induced by the sustained tactile stimuli further confirmed that the stimulusinduced oscillatory dynamic may be considered a novel brain signal modality for tactile BCI research, which significantly enhanced the performance of the SAO-based BCI [27].

The current study was partly motivated by our previous study [43], in which the concept of tendon vibration to induce sensation with kinesthesia illusion was for the first time introduced to enhance MI-based BCI system. We proposed a stimulation assisted training paradigm, in which every illusory sensation trial was followed by a motor imagery trial, and we have shown that this provides a way to improve MI performance in a BCI setting. By contrast, based on the similarity between tactile sensation and imagined sensation, we have found that the sensory stimulation provided a new approach to further improve SAO-based BCI performance. The experimental protocol was different from our previous study. Here, instead of an alternation between SS and SAO [43], the subjects received only real tactile sensation during the second block, while during the last block subjects only performed imagined sensation tasks. Moreover, the stimulation types differed, since in our previous study illusory movement was induced via tendon stimulation, which required a much stronger mechanical stimulation that elicited several types of sensory receptors (Group Ia, II and Ib, likely also joint receptors). In the current study, the stimulus was targeted at the tactile sensation level.

MI and SAO are both based on the dynamics of brain oscillation quantified as ERD/ERS [48], [53], which have a strong correlation not only with real or imagined movement [2], [54], [55], but also with sensory processing [56]-[59], or even imagined sensation [32]. Previous studies have shown that the combination of $\mathrm{MI}$ and SAO results in a significantly improved BCI performance [26], [33]. As for the activation of similar brain areas for real versus imagined movements, the current results confirm that brain activity patterns are also similar between real and imagined sensation in the contex of ERD/ERS dynamics [32]. One is externally induced (SS), while the other is internally generated (SAO). Such similarity of the EDR/ERS characteristics between SS and SAO provides a novel way to train subjects to achieve the initial control of a SAO-based BCI, 
which is independent of external stimuli. Our recent findings have shown that the covert SAO intention may be reliably decoded by a BCI system calibrated with SS [44]. The SST training protocol would establish a novel framework to improve SAO-based BCI performance, independent of specific algorithms used. The advanced algorithms developed for MIbased BCIs might also work well in SAO-based BCI. Combing the proposed sensory stimulation training with advanced algorithms would potentially further improve SAO-based BCI performance, which will be investigated in future studies.

In the current study, the concept of SST training was proposed to facilitate somatosensory BCI performance. One limitation of the current study is that only the short-term training effect was evaluated, as only two runs ( 80 trials) were performed in the last block. For a practical BCI application, the long-term training effect, or the performance changes across a longer time frame (hours or days) should be further explored. In current study, the statistics did not reveal a significant difference between the SST-Group and the Control-Group in the first block, although they were some noticeable differences. One of the reason for such differences is likely the variance among subjects, as exhibited in the literature and also shown in our previous study [34]. In the current study, we focused on the change (both BCI performance and ERD/ERS dynamics) after the SST training, and the control group was mainly used as a control to indicate no learning effect existed in current threeblock design. The improvement of the BCI performance may thus be related to the SST training and not any learning effect.

\section{CONCLUSION}

In this study, sensory stimulation training (SST) was proposed to improve the performance of a SAO-based BCI system. We demonstrated that SST training significantly improved the SAO-based BCI by $9.4 \%$ while no significant difference was found in the Control-Group. The proposed SST thus provides a new training framework to further improve BCI performance in the decoding of somatosensory attention.

\section{ACKNOWLEDGEMENT}

We thank all volunteers for their participation in this study. This work is supported by the University Starter Grant of the University of Waterloo (No. 203859), the National Natural Science Foundation of China (Grant No. 51620105002).

\section{REFERENCE}

J. R. Wolpaw, N. Birbaumer, D. J. McFarland, G. Pfurtscheller, T. M. Vaughan, and others, "Brain-computer interfaces for communication and control," Clin. Neurophysiol., vol. 113, no. 6, pp. 767-791, 2002.

[2] G. Pfurtscheller, C. Neuper, and others, "Motor imagery activates primary sensorimotor area in humans.," Neurosci. Lett., vol. 239, no. 2-3, p. 65, 1997.

[3] G. Pfurtscheller and C. Neuper, "Motor imagery and direct braincomputer communication," Proc. IEEE, vol. 89, no. 7, pp. 11231134, 2001.

[4] S. N. Baker, "Oscillatory interactions between sensorimotor cortex and the periphery," Curr. Opin. Neurobiol., vol. 17, no. 6, pp. 649655, Dec. 2007.

[5] Han Yuan and Bin $\mathrm{He}$, "Brain-Computer Interfaces Using
Sensorimotor Rhythms: Current State and Future Perspectives," IEEE Trans. Biomed. Eng., vol. 61, no. 5, pp. 1425-1435, 2014.

[6] G. Pfurtscheller, "Spatiotemporal ERD/ERS patterns during voluntary movement and motor imagery," Suppl. Clin. Neurophysiol., vol. 53, pp. 196-198, 2000.

[7] G. Pfurtscheller, C. Brunner, A. Schlögl, and F. H. Lopes da Silva, "Mu rhythm (de)synchronization and EEG single-trial classification of different motor imagery tasks," Neuroimage, vol. 31, no. 1, pp. 153-159, 2006.

[8] R. Xu, N. Jiang, C. Lin, N. Mrachacz-Kersting, K. Dremstrup, and D. Farina, "Enhanced low-latency detection of motor intention from EEG for closed-loop brain-computer interface applications," IEEE Trans. Biomed. Eng., vol. 61, no. 2, pp. 288-296, Feb. 2014

[9] I. K. Niazi, N. Jiang, O. Tiberghien, J. F. Nielsen, K. Dremstrup, and D. Farina, "Detection of movement intention from single-trial movement-related cortical potentials.," J. Neural Eng., vol. 8, no. 6, p. 66009 , Dec. 2011.

[10] B. Blankertz et al., "The BCI competition 2003: progress and perspectives in detection and discrimination of EEG single trials," IEEE Trans. Biomed. Eng., vol. 51, no. 6, pp. 1044-1051, 2004

[11] B. Blankertz et al., "The BCI competition III: Validating alternative approaches to actual BCI problems," IEEE Trans. Neural Syst. Rehabil. Eng., vol. 14, no. 2, pp. 153-159, 2006.

[12] M. Tangermann et al., "Review of the BCI competition IV," Front. Neurosci., vol. 6, 2012.

[13] J. Meng, L. Yao, X. Sheng, D. Zhang, and X. Zhu, "Simultaneously optimizing spatial spectral features based on mutual information for EEG classification," IEEE Trans. Biomed. Eng., vol. 62, no. 1, pp. 227-240, 2015

[14] J. Long, Y. Li, H. Wang, T. Yu, J. Pan, and F. Li, “A Hybrid Brain Computer Interface to Control the Direction and Speed of a Simulated or Real Wheelchair," Neural Syst. Rehabil. Eng. IEEE Trans., vol. 20, no. 5, pp. 720-729, Sep. 2012.

[15] A. R. Architecture, "Brain-Controlled Wheelchairs," no. March, pp. 65-73, 2013.

[16] A. J. Doud, J. P. Lucas, M. T. Pisansky, and B. He, "Continuous three-dimensional control of a virtual helicopter using a motor imagery based brain-computer interface," PLoS One, vol. 6, no. 10, p. e26322, 2011.

[17] K. LaFleur, K. Cassady, A. Doud, K. Shades, E. Rogin, and B. He, "Quadcopter control in three-dimensional space using a noninvasive motor imagery-based brain-computer interface.," J. Neural Eng., vol. 10, no. 4, p. 46003, Aug. 2013.

[18] J. Meng, S. Zhang, A. Bekyo, J. Olsoe, B. Baxter, and B. He, "Noninvasive Electroencephalogram Based Control of a Robotic Arm for Reach and Grasp Tasks," Sci. Rep., vol. 6, no. November, p. $38565,2016$.

[19] G. Pfurtscheller, G. R. Müller, J. Pfurtscheller, H. J. Gerner, and R. Rupp, “'Thought'--control of functional electrical stimulation to restore hand grasp in a patient with tetraplegia," Neurosci. Lett., vol. 351, no. 1, pp. 33-36, 2003.

[20] G. R. Müller-Putz, R. Scherer, G. Pfurtscheller, and R. Rupp, "EEGbased neuroprosthesis control: A step towards clinical practice," Neurosci. Lett., vol. 382, no. 1-2, pp. 169-174, 2005.

[21] K. K. Ang and C. Guan, "Brain-computer interface for neurorehabilitation of upper limb after stroke," Proc. IEEE, vol. 103, no. 6, pp. 944-953, 2015.

[22] F. Pichiorri, N. Mrachacz-Kersting, M. Molinari, S. Kleih, A. Kübler, and D. Mattia, "Brain-computer interface based motor and cognitive rehabilitation after stroke - state of the art, opportunity, and barriers: summary of the BCI Meeting 2016 in Asilomar," Brain-Computer Interfaces, vol. 4, no. 1-2, pp. 53-59, 2017.

[23] S. Silvoni et al., "Brain-Computer Interface in Stroke: A Review of Progress," Clin. EEG Neurosci., vol. 42, no. 4, pp. 245-252, 2011.

[24] A. Ramos-Murguialday et al., "Brain-machine interface in chronic stroke rehabilitation: A controlled study," Ann. Neurol., vol. 74, no. 1, pp. 100-108, 2013.

[25] L. Yao, J. Meng, D. Zhang, X. Sheng, and X. Zhu, "Selective Sensation Based Brain-Computer Interface via Mechanical Vibrotactile Stimulation," PLoS One, vol. 8, no. 6, p. e64784, 2013.

[26] L. Yao, J. Meng, D. Zhang, X. Sheng, and X. Zhu, "Combining motor imagery with selective sensation toward a hybrid-modality BCI," IEEE Trans. Biomed. Eng., vol. 61, no. 8, pp. 2304-2312, 2014.
L. Yao et al., "A Multi-Class Tactile Brain-Computer Interface Based on Stimulus-Induced Oscillatory Dynamics," IEEE Trans. 
[28]

Neural Syst. Rehabil. Eng., vol. 26, no. 1, pp. 3-10, Jan. 2018. G. R. Müller-Putz, R. Scherer, C. Neuper, and G. Pfurtscheller, "Steady-state somatosensory evoked potentials: Suitable brain signals for brain-computer interfaces?," IEEE Trans. Neural Syst. Rehabil. Eng., vol. 14, no. 1, pp. 30-37, 2006.

[29] S. Ahn, K. Kim, and S. C. Jun, "Steady-State Somatosensory Evoked Potential for Brain-Computer Interface--Present and Future," Front. Hum. Neurosci., vol. 9, p. 716, 2015.

[30] S. Ahn, M. Ahn, H. Cho, S. Chan Jun, and S. C. Jun, "Achieving a hybrid brain-computer interface with tactile selective attention and motor imagery,” J. Neural Eng., vol. 11, no. 6, p. 66004, Dec. 2014.

[31] A.-M. Brouwer and J. B. F. Van Erp, "A tactile P300 brain-computer interface," Front. Neurosci., vol. 4, p. 19, 2010.

[32] L. Yao, X. Sheng, D. Zhang, N. Jiang, D. Farina, and X. Zhu, "A BCI System based on Somatosensory Attentional Orientation," IEEE Trans. Neural Syst. Rehabil. Eng., vol. 4320, no. c, pp. 1-1, Jan. 2016.

[33] L. Yao et al., "A Stimulus-Independent Hybrid BCI Based on Motor Imagery and Somatosensory Attentional Orientation," IEEE Trans. Neural Syst. Rehabil. Eng., vol. 25, no. 9, pp. 1674-1682, 2017.

[34] L. Yao, X. Sheng, N. Mrachacz-Kersting, X. Zhu, D. Farina, and N. Jiang, "Performance of Brain-Computer Interfacing Based on Tactile Selective Sensation and Motor Imagery," IEEE Trans. Neural Syst. Rehabil. Eng., vol. 4320, no. c, pp. 1-1, 2017.

[35] V. Kaiser et al., "Cortical effects of user training in a motor imagery based brain-computer interface measured by fNIRS and EEG.," Neuroimage, vol. 85 Pt 1, pp. 432-44, Jan. 2014.

[36] F. Pichiorri et al., "Sensorimotor rhythm-based brain-computer interface training: the impact on motor cortical responsiveness.," $J$. Neural Eng., vol. 8, no. 2, p. 25020, Apr. 2011.

[37] J. R. Wolpaw and D. J. McFarland, "Control of a two-dimensional movement signal by a noninvasive brain-computer interface in humans," Proc. Natl. Acad. Sci. U. S. A., vol. 101, no. 51, pp. 17849 17854, 2004.

[38] C. Neuper and G. Pfurtscheller, "Neurofeedback training for BCI control," in Brain-Computer Interfaces, Springer, 2009, pp. 65-78.

[39] C. Neuper, A. Schlögl, and G. Pfurtscheller, "Enhancement of leftright sensorimotor EEG differences during feedback-regulated motor imagery," J. Clin. Neurophysiol., vol. 16, no. 4, pp. 373-382, 1999.

[40] F. Cincotti et al., "Vibrotactile feedback for brain-computer interface operation.," Comput. Intell. Neurosci., vol. 2007, p. 48937, Jan. 2007.

[41] M. Pham et al., "An auditory brain-computer interface based on the self-regulation of slow cortical potentials," Neurorehabil. Neural Repair, vol. 19, no. 3, pp. 206-218, 2005.

[42] C. Vidaurre, C. Sannelli, K.-R. Müller, and B. Blankertz, "Coadaptive calibration to improve BCI efficiency," J. Neural Eng., vol. 8, no. 2, p. 25009,2011

[43] L. Yao, J. Meng, X. Sheng, D. Zhang, and X. Zhu, "A novel calibration and task guidance framework for motor imagery BCI via a tendon vibration induced sensation with kinesthesia illusion.," $J$. Neural Eng., vol. 12, no. 1, p. 16005, Dec. 2015.

[44] L. Yao, X. Sheng, N. Mrachacz-Kersting, X. Zhu, D. Farina, and N. Jiang, "Decoding covert somatosensory attention by a BCI system calibrated with tactile sensation," IEEE Trans. Biomed. Eng., vol.
9294, no. c, pp. 1-1, 2017.

[45] C. Breitwieser, V. Kaiser, C. Neuper, and G. R. Müller-Putz, "Stability and distribution of steady-state somatosensory evoked potentials elicited by vibro-tactile stimulation," Med. Biol. Eng. Comput., pp. 1-11, 2012.

[46] H. Ramoser, J. Muller-Gerking, and G. Pfurtscheller, "Optimal spatial filtering of single trial EEG during imagined hand movement," IEEE Trans. Rehabil. Eng., vol. 8, no. 4, pp. 441-446, 2000.

[47] B. Blankertz, R. Tomioka, S. Lemm, M. Kawanabe, and K.-R. Muller, "Optimizing spatial filters for robust EEG single-trial analysis," IEEE Signal Process. Mag., vol. 25, no. 1, pp. 41-56, 2008.

[48] B. Graimann, J. E. Huggins, S. P. Levine, and G. Pfurtscheller, "Visualization of significant ERD/ERS patterns in multichannel EEG and ECoG data," Clin. Neurophysiol., vol. 113, no. 1, pp. 43-47, 2002.

[49] A. Delorme et al., "EEGLAB, SIFT, NFT, BCILAB, and ERICA: new tools for advanced EEG processing," Comput. Intell. Neurosci., vol. 2011, p. 10, 2011.

[50] R. Oostenveld, P. Fries, E. Maris, and J. M. Schoffelen, "FieldTrip: open source software for advanced analysis of MEG, EEG, and invasive electrophysiological data," Comput. Intell. Neurosci., vol. 2011, p. 1, 2011.

[51] T. H. Wonnacott and R. J. Wonnacott, Introductory statistics, vol. 19690. Wiley Chichester, 1990.

[52] G. Schalk, D. J. McFarland, T. Hinterberger, N. Birbaumer, and J. R. Wolpaw, "BCI2000: a general-purpose brain-computer interface (BCI) system," IEEE Trans. Biomed. Eng., vol. 51, no. 6, pp. 1034 1043, 2004.

[53] G. Pfurtscheller and F. H. da Silva, "Event-related EEG/MEG synchronization and desynchronization: basic principles," Clin. Neurophysiol., vol. 110, no. 11, pp. 1842-1857, 1999.

[54] F. Cassim et al., "Brief and sustained movements: differences in event-related (de) synchronization (ERD/ERS) patterns," Clin. Neurophysiol., vol. 111, no. 11, pp. 2032-2039, 2000.

[55] G. Pfurtscheller, C. Neuper, C. Brunner, F. L. da Silva, and others, "Beta rebound after different types of motor imagery in man.," Neurosci. Lett., vol. 378, no. 3, p. 156, 2005.

[56] N. Sharma, V. M. Pomeroy, and J.-C. Baron, "Motor imagery: a backdoor to the motor system after stroke?," Stroke., vol. 37, no. 7, pp. 1941-52, Jul. 2006.

[57] E. Houdayer et al., "Movement preparation and cortical processing of afferent inputs in cortical tremor: an event-related (de)synchronization (ERD/ERS) study.," Clin. Neurophysiol., vol. 123, no. 6, pp. 1207-15, Jun. 2012.

[58] G. Pfurtscheller et al., "Contrasting behavior of beta event-related synchronization and somatosensory evoked potential after median nerve stimulation during finger manipulation in man.," Neurosci. Lett., vol. 323, no. 2, p. 113, 2002.

[59] E. Houdayer, E. Labyt, F. Cassim, J. L. Bourriez, and P. Derambure, "Relationship between event-related beta synchronization and afferent inputs: analysis of finger movement and peripheral nerve stimulations.," Clin. Neurophysiol., vol. 117, no. 3, pp. 628-36, Mar. 2006 